\title{
Análisis de integrales trigonométricas notables
}

\author{
DOI: $\underline{\text { https://doi.org/10.33262/ap.v3i3.1.89 }}$
}

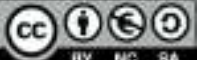

\section{Analysis of notable trigonometric integrals}

Rómel Manolo Insuasti Castelo. ${ }^{1} \&$ Javier Roberto Mendoza Castillo. ${ }^{2}$

\begin{abstract}
.
Introduction. The calculation of trigonometric integrals is a process that involves the use of some integration techniques, for this you must be clear about the procedures to follow and be very observant when choosing the appropriate integration technique Objective. We want to simplify the calculation of the notable trigonometric integral. Methodology. To understand this methodology, it is necessary to know some concepts that are around the trigonometric functions to be integrated, such as the periodicity of a function, its parity (even or odd function), and that the integrals are evaluated in the limits corresponding to the intervals of the periodicity, we will call this type of integrals notable integrals since it is not strictly necessary to evaluate them by integration processes but rather their result is deduced from the aforementioned concepts. Results. Although it is true that the aforementioned integrals can be found solved, the intention of this analysis is to show that the result of these integrals is easy to remember in communion with the concepts mentioned in a mechanical and simple way.
\end{abstract}

Keywords: Trigonometric integrals, periodic functions, odd and even functions.

\section{Resumen.}

Introducción. El cálculo de integrales trigonométricas es un proceso que reviste el empleo de algunas técnicas de integración, para esto se debe tener claro los

1 Escuela Superior Politécnica de Chimborazo, Facultad de Mecánica. Riobamba. Ecuador. rinsuasti@espoch.edu.ec. Código ORCID: https://orcid.org/0000-0002-4170-1511

2 Escuela Superior Politécnica de Chimborazo, Facultad de Recursos Naturales. Riobamba, Ecuador. jmendoza@espoch.edu.ec. Código ORCID: https://orcid.org/0000-0003-3148-0193 
procedimientos a seguir y ser muy observador al elegir la técnica de integración apropiada. Objetivo. Se desea simplificar el cálculo de la integral trigonométrica notable. Metodología. Para entender esta metodología es necesario conocer algunos conceptos que están alrededor de las funciones trigonométricas a integrar, como son la periodicidad de una función, su paridad (función par o impar), y que las integrales estén evaluadas en los límites correspondientes a los intervalos de la periodicidad, a este tipo de integrales las denominaremos integrales notables pues no es estrictamente necesario evaluarlas por procesos de integración sino que se deducen su resultado a partir de los conceptos antes mencionados. Resultados. Si bien es cierto que las integrales mencionadas se las pueden encontrar resueltas, la intención de este análisis es mostrar que el resultado de estas integrales es fácil recordar en comunión de los conceptos citados de manera mecánica y sencilla.

Palabras claves: Integrales trigonométricas, funciones periódicas, funciones pares e impares

\section{Introducción.}

La solución de integrales definidas de funciones trigonométricas en ocasiones se encuentra en el desarrollo de problemas de diferente índole matemático, por lo que es necesario resolverlas siendo en ocasiones innecesario, pues si se analiza un poco, estas integrales se las puede resolver por simple inspección llegándose a determinar que dichas integrales son notables, es decir que no requieren un proceso de solución, para determinar el valor de dicha integral. Para esto se requiere el conocimiento de ciertos conceptos trigonométricos y del análisis matemático. Algunos conceptos a tener en cuenta son el de función par e impar, función periódica, integral definida, identidades trigonométricas, entre otros los que permitirán analizar la integral en su totalidad, dichas integrales tienen ciertas condiciones que determinan el valor de las mismas, sin calcularlas. (Araujo, 2018). La utilidad de esas funciones se encuentra en las aplicaciones de las series de Fourier (Villalobos, 2011) en donde se encuentran funciones con las características que se pretende estudiar en este artículo. Poniendo en evidencia la aplicación de los conceptos antes mencionados.

\section{Metodología.}

Revisemos algunos conceptos que se encuentran en la mayoría de bibliografía matemática, así citaremos a Leithold, Araujo, Purcell, Stewart y Apostol muy conocidos en el Ecuador.

Función par: Se dice que una función es par si se cumple la siguiente igualdad

$$
f(x)=f(-x)
$$


Esta ecuación nos indica que para cualquier valor de $x$ y $-x$ el valor de la función $f(x)$, es el mismo valor, también podríamos decir que la función es simétrica al eje y (Espinoza, 2005).

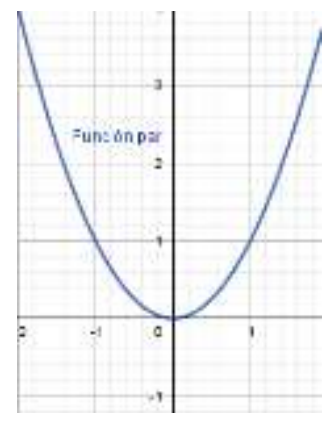

Gráfico 1. Función par

Fuente: Elaboración propia

Función impar: Se dice que una función es impar si se cumple la siguiente igualdad

$$
f(-x)=-f(x)
$$

Esta ecuación nos indica que para cualquier valor de $x$ y $-x$ el valor de la función $f(x)$, es el mismo valor pero de signo contrario, también podríamos decir que la función no es simétrica al eje $\mathrm{y}$.

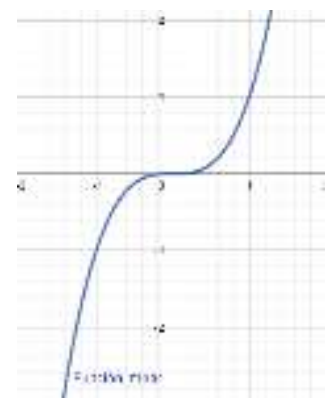

Gráfico 2. Función impar

Fuente: Elaboración propia

Periodicidad de una función.

La periodicidad de una función debe entenderse como el hecho de que gráficamente la función se repite cada cierto valor de $\mathrm{x}$, y este representa un intervalo sobre el eje $\mathrm{x}$, matemáticamente se lo puede definir como:

$$
f(x)=f(x+n p)
$$

Donde $\mathrm{p}$, es periodo de la función, este valor puede ser un múltiplo $\mathrm{n}$ de $\mathrm{p}, \mathrm{n}$ es un escalar, tal como p, 2 p, 3 p, etc., estos indican que también son periodos de $f(x)$.

Las funciones trigonométricas son funciones periódicas (Lehmann, 1989), como podemos ver en el gráfico 3 y gráfico 4 . 


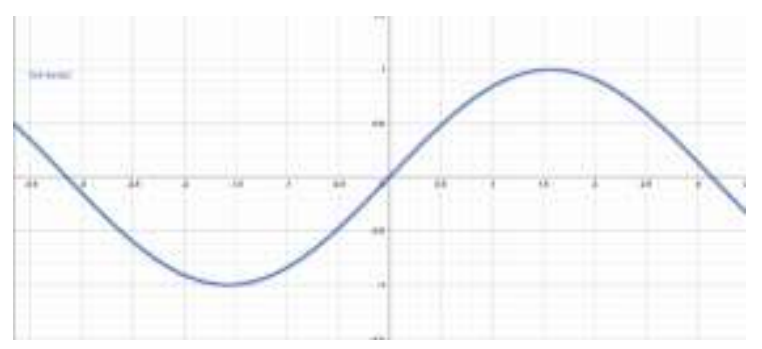

Gráfico 3. Función $\sin (\mathrm{x})$

Fuente: Elaboración propia

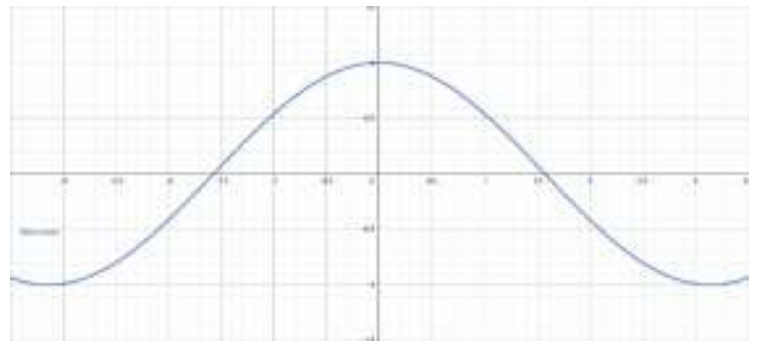

Gráfico 4. Función cos $(x)$

Fuente: Elaboración propia

Integral definida: La interpretación geométrica de una integral definida es el área bajo la curva en un intervalo respecto de la variable independiente de la función analizada, donde el área sobre el eje se considera área positiva y el área debajo del eje se considera área negativa como se observa en el Gráfico 4.

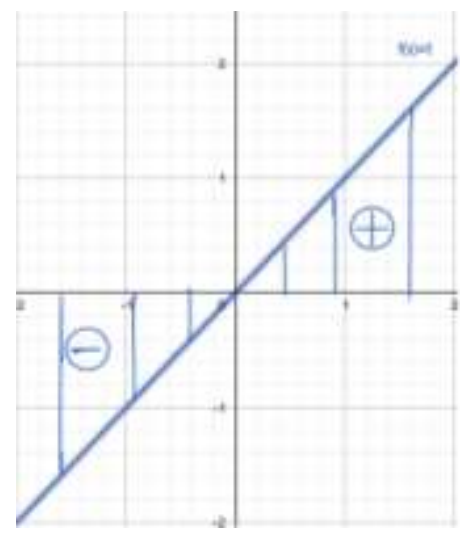

Gráfico 5. Interpretación geométrica de la integral definida

Fuente: Elaboración propia

Citamos a Niles que trata algunas identidades trigonométricas que encontramos muy útiles, en la integración de funciones trigonométricas:

$$
\begin{aligned}
& \sin \alpha \pm \sin \beta=2 \cdot \sin \frac{\alpha \pm \beta}{2} \cdot \cos \frac{\alpha \mp \beta}{2} \\
& \cos \alpha+\cos \beta=2 \cdot \cos \frac{\alpha+\beta}{2} \cdot \cos \frac{\alpha-\beta}{2}
\end{aligned}
$$




$$
\begin{gathered}
\cos \alpha-\cos \beta=-2 \cdot \sin \frac{\alpha+\beta}{2} \cdot \sin \frac{\alpha-\beta}{2} \\
\sin ^{2} \alpha=\frac{1-\cos 2 \alpha}{2} \\
\cos ^{2} \alpha=\frac{\cos 2 \alpha+1}{2}
\end{gathered}
$$

\section{Análisis.}

Las integrales a ser analizadas son de funciones trigonométricas, periódicas en $2 \pi$ donde los límites de integración están en el intervalo de $2 \pi$. (Salinas, 2011)

a. Sea la integral $\int_{-\pi}^{\pi} \sin (n x) d x$, la función $\sin (n x)$, es una función impar y periódica de lo cual se deduce por la integral en el intervalo de que existe un área sobre el eje que es igual al área debajo del eje de lo cual se deduce por simple inspección que el área total en el intervalo del periodo es cero, como podemos observar en la Gráfica 5

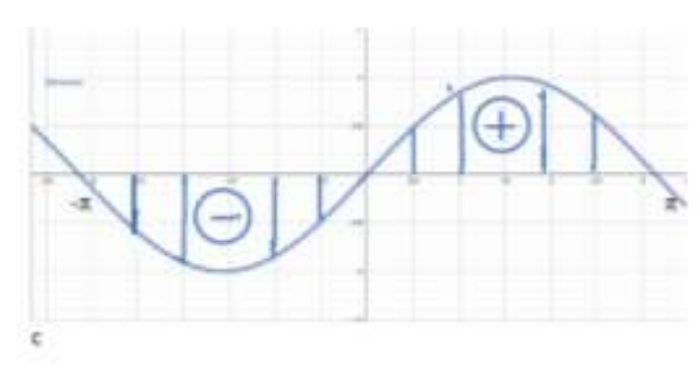

Gráfico 6. Integral definida del $\sin (\mathrm{x})$

Fuente: Elaboración propia

Por lo tanto: $\int_{-\pi}^{\pi} \sin (n x) d x=0 \quad$ constituyéndose en una integral notable.

b. De forma análoga podemos realizar la integral $\int_{-\pi}^{\pi} \cos (n x) d x$, la función es par y periódica, la cual al analizar la curva se puede observar que las áreas de la curva en el periodo suman cero, por lo que su integral se calculará por simple inspección.

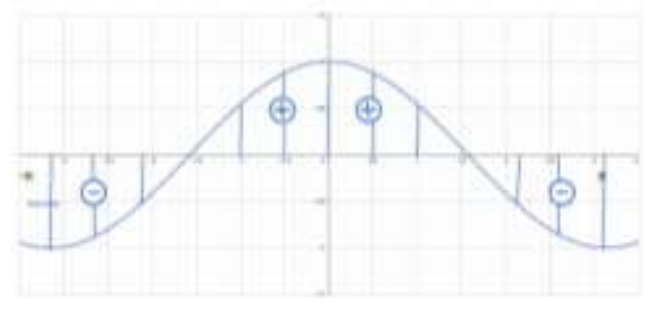

Gráfico 7. Integral definida del $\cos (x)$

Fuente: Elaboración propia 
Por lo tanto:

$$
\int_{-\pi}^{\pi} \cos (n x) d x=0
$$

Estas dos integrales nos servirán como base para trabajar con otras integrales un poco más complejas.

c. Sea la integral:

$$
\int_{-\pi}^{\pi} \cos (n x) \sin (m x) d x
$$

Como se observa la función de la integral $\cos (n x) \sin (m x)$ puede ser remplazada por la identidad trigonométrica: (Ellis, 2006)

$$
\sin \alpha \pm \sin \beta=2 \cdot \sin \frac{\alpha \pm \beta}{2} \cdot \cos \frac{\alpha \mp \beta}{2}
$$

En la que se puede observar, que se puede realizar un cambio en la función de un producto de un seno por un coseno a una suma de senos, teniendo en cuenta que:

$$
\begin{aligned}
& \frac{\alpha \mp \beta}{2}=n x \\
& \frac{\alpha \pm \beta}{2}=m x
\end{aligned}
$$

Al cambiar a la suma de senos esto implica tener integrales de senos, que como se ha visto estas son cero, por lo tanto se puede afirmar sin importar cuales sean los valores de m y n, que:

$$
\int_{-\pi}^{\pi} \cos (n x) \sin (m x) d x=0
$$

d. Sea la integral:

$$
\int_{-\pi}^{\pi} \cos (n x) \cos (m x) d x
$$

En este caso tenemos dos posibilidades de análisis, dependiendo de los valores de $\mathrm{m}$ y n, así:

Si $m \neq n$ entonces:

$$
\int_{-\pi}^{\pi} \cos (n x) \cos (m x) d x=0
$$

De acuerdo a la identidad trigonométrica: 


$$
\cos \alpha+\cos \beta=2 \cdot \cos \frac{\alpha+\beta}{2} \cdot \cos \frac{\alpha-\beta}{2}
$$

Podemos observar que se puede transformar el producto de dos cosenos en la suma de cosenos, teniendo en cuenta que

$$
\begin{aligned}
& \frac{\alpha+\beta}{2}=n x \\
& \frac{\alpha-\beta}{2}=m x
\end{aligned}
$$

Por lo tanto las integrales de cosenos sabemos que son cero y el resultado entonces será:

$$
\int_{-\pi}^{\pi} \cos (n x) \cos (m x) d x=0
$$

Si $m=n$, entonces en la integral se tendría

$$
\int_{-\pi}^{\pi} \cos (n x) \cos (n x) d x=\int_{-\pi}^{\pi} \cos ^{2}(n x) d x
$$

Al remplazar por la identidad trigonométrica

$$
\cos ^{2} \alpha=\frac{\cos 2 \alpha+1}{2}
$$

Se tiene

$$
\int_{-\pi}^{\pi} \frac{\cos 2 x+1}{2} d x
$$

Abriendo la integral se tiene:

$$
\frac{1}{2} \int_{-\pi}^{\pi} \cos 2 x d x+\frac{1}{2} \int_{-\pi}^{\pi} d x
$$

Donde la integral de coseno es cero y la segunda integral al evaluarla es igual a $\pi$, por lo tanto:

$$
\int_{-\pi}^{\pi} \cos (n x) \cos (m x) d x=\pi \text { si } m=n
$$

e. Sea la integral:

$$
\int_{-\pi}^{\pi} \sin (n x) \sin (m x) d x
$$


En forma análoga en este caso tenemos dos posibilidades de análisis, dependiendo de los valores de m y n, así:

Si $m \neq n$ entonces:

$$
\int_{-\pi}^{\pi} \sin (n x) \sin (m x) d x=0
$$

De acuerdo a la identidad trigonométrica:

$$
\cos \alpha-\cos \beta=-2 \cdot \sin \frac{\alpha+\beta}{2} \cdot \sin \frac{\alpha-\beta}{2}
$$

Podemos observar que se puede transformar el producto de dos senos en la diferencia de cosenos, teniendo en cuenta que

$$
\begin{aligned}
& \frac{\alpha+\beta}{2}=n x \\
& \frac{\alpha-\beta}{2}=m x
\end{aligned}
$$

Por lo tanto las integrales de cosenos sabemos que son cero y el resultado entonces será:

$$
\int_{-\pi}^{\pi} \cos (n x) \cos (m x) d x=0
$$

Si $m=n$, entonces en la integral se tendría

$$
\int_{-\pi}^{\pi} \sin (n x) \sin (n x) d x=\int_{-\pi}^{\pi} \sin ^{2}(n x) d x
$$

Al remplazar por la identidad trigonométrica

$$
\sin ^{2} \alpha=\frac{1-\cos 2 \alpha}{2}
$$

Se tiene

$$
\int_{-\pi}^{\pi} \frac{1-\cos 2 \alpha}{2} d x
$$

Abriendo la integral se tiene:

$$
\frac{1}{2} \int_{-\pi}^{\pi} d x-\frac{1}{2} \int_{-\pi}^{\pi} \cos 2 x d x
$$

Donde la integral de coseno es cero y la primera integral al evaluarla es igual a $\pi$, por lo tanto: 


$$
\int_{-\pi}^{\pi} \sin (n x) \sin (m x) d x=\pi \quad \text { si } m=n
$$

\section{Resultados.}

Como se puede observar se ha podido diseñar y definir integrales que son de fácil evaluación (Favieri, 2009), si se hace uso de los conceptos matemáticos logrando obtener integrales notables de funciones trigonométricas evaluadas en un intervalo que corresponde al periodo de la función, las cuales se resumen en la siguiente tabla.

Tabla 1

Integrales Notables

\begin{tabular}{|c|c|c|c|}
\hline & Integrales notables & Valor & Condición \\
\hline $\mathbf{a}$ & $\int_{-\pi} \sin (n x) d x$ & 0 & \\
\hline b & $\int_{-\pi}^{\pi} \cos (n x) d x$ & 0 & \\
\hline c & $\int_{-\pi}^{\pi} \cos (n x) \sin (m x) d x$ & 0 & \\
\hline \multirow{2}{*}{ d } & \multirow{2}{*}{$\int_{-\pi}^{\pi} \cos (n x) \cos (m x) d x$} & 0 & $m \neq n$ \\
\hline & & $\pi$ & $\mathrm{Si} \quad m=n$ \\
\hline \multirow{2}{*}{$\mathbf{e}$} & \multirow{2}{*}{$\int_{-\pi}^{\pi} \sin (n x) \sin (m x) d x$} & 0 & $\mathrm{Si} \quad m \neq n$ \\
\hline & & $\pi$ & $\mathrm{Si} \quad m=n$ \\
\hline
\end{tabular}

\section{Conclusiones.}

- Se pude aceptar el término de integrales trigonométricas notables, a aquellas integrales a las cuales se las puede obtener el resultado de una forma mecanizada y ordenada basada en principios matemáticos, como son funciones periódicas, funciones pares o impares, e integrales evaluadas en el intervalo de la periodicidad, lo que evita encontrar la integral a través de procesos de integración.

- El valor de las integrales del seno como del coseno es igual a cero siempre y cuando sean evaluados dentro del intervalo de la periodicidad de las mismas y se constituyen en la base para encontrar las demás integrales notables.

- Los valores de las integrales de la tabla de resumen quedan a disposición y pueden ser base para el cálculo de otras integrales.

\section{Referencias bibliográficas.}

Apostol, T. M. (2005). Calculus, Vol. 1, Reverté, 2a . Edición, 11-15

Araujo, F. (2018). Cálculo integral. Quito: Editorial Universitaria Abya-Yala.,19-22 
Ellis, R And Gulick D. (2006). Calculus W/Concepts in Calculus. Sixth Edition. Editorial: Cengage Learning,125-129

Espinoza, E (2005), Matemática Básica, 2ª Edición, Perú., 339-340

Favieri, A., \& Scorzo, R. (2009). Estudio sobre habilidades matemáticas para el Cálculo Diferencial en estudiantes de Ingeniería. . 10mo Simposio De Educación Matemática.,4-5

Lehmann, Ch. (1989), Geometría Analítica, 13a ${ }^{\text {a }}$ Edición. México D.F., Limusa., 298 300

Leithold L., El cálculo. 7ª ed. México: Printed Oxford University Press-Harla México, S. A. de C. V., 1998. 242-248

Niles O. N. (1988). Trigonometría Plana. Limusa, S. A de C. V., México D. F. , 127-147.

Purcell E., Verberg D. y Rigdon S., Cálculo, 9ª . ed. México: Pearson Educación, 2007., 215-224

Salinas, G (2011), Algebra Superior, $1^{\text {a }}$ Edición, Ecuador, Soluciones Gráficas., 44-46

Stewart, J. (2018). Cálculo de una variable. Trascendentes tempranas. Cengage, 8a. Edición, 2018., 360-369

Villalobos, A. y García, G.(2011) Algunas Series E Integrales Con Funciones Trigonométricas Centro de Investigación de Matemática Aplicada (C.I.M.A.),4-6

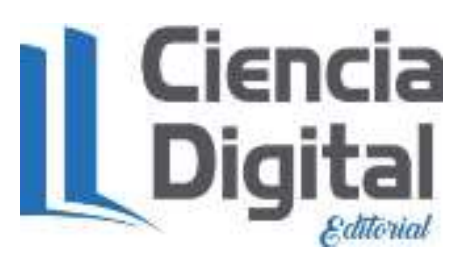




\section{PARA CITAR EL ARTÍCULO INDEXADO.}

Insuasti Castelo, R. M., \& Mendoza Castillo, J. R. (2021). Análisis de integrales trigonométricas notables . AlfaPublicaciones, 3(3.1), 238-248. https://doi.org/10.33262/ap.v3i3.1.89

\section{Ciencia}

El artículo que se publica es de exclusiva responsabilidad de los autores y no necesariamente reflejan el pensamiento de la Revista Alfa Publicaciones.

El artículo queda en propiedad de la revista y, por tanto, su publicación parcial y/o total en otro medio tiene que ser autorizado por el director de la Revista Alfa Publicaciones.
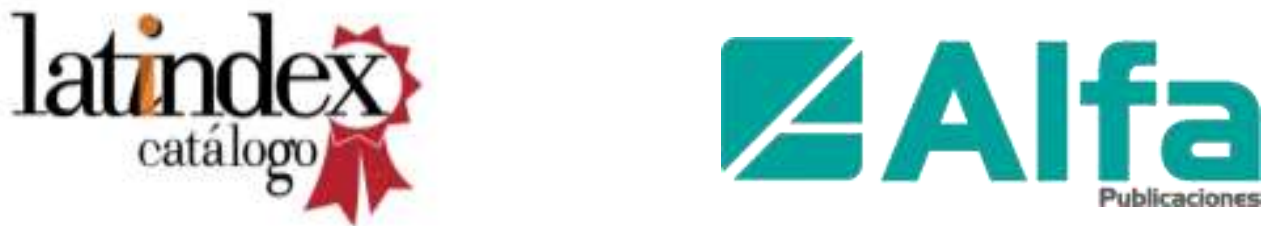